\title{
An Auditory Go/No-Go Study of Event-Related Potentials in Children with Fetal Alcohol Spectrum Disorders
}

Steinmann, Tobias P.; Andrew, Colin M.; Thomsen, Carsten E.; Kjær, Troels W.; Meintjes, Ernesta M.; Molteno, Christopher D.; Jacobson, Joseph L.; Jacobson, Sandra W.; Sørensen, Helge Bjarup Dissing

Published in:

IEEE Engineering in medicine and biology society conference proceedings

Link to article, DOI:

10.1109/IEMBS.2011.6090181

Publication date:

2011

Link back to DTU Orbit

Citation $(A P A)$ :

Steinmann, T. P., Andrew, C. M., Thomsen, C. E., Kjær, T. W., Meintjes, E. M., Molteno, C. D., Jacobson, J. L., Jacobson, S. W., \& Sørensen, H. B. D. (2011). An Auditory Go/No-Go Study of Event-Related Potentials in Children with Fetal Alcohol Spectrum Disorders. In IEEE Engineering in medicine and biology society conference proceedings (pp. 789-792). IEEE. https://doi.org/10.1109/IEMBS.2011.6090181

\section{General rights}

Copyright and moral rights for the publications made accessible in the public portal are retained by the authors and/or other copyright owners and it is a condition of accessing publications that users recognise and abide by the legal requirements associated with these rights.

- Users may download and print one copy of any publication from the public portal for the purpose of private study or research.

- You may not further distribute the material or use it for any profit-making activity or commercial gain

- You may freely distribute the URL identifying the publication in the public portal 


\title{
An Auditory Go/No-Go Study of Event-Related Potentials in Children with Fetal Alcohol Spectrum Disorders
}

\author{
Tobias P. Steinmann*, Colin M. Andrew ${ }^{\dagger}$, Carsten E. Thomsen ${ }^{\dagger}$, Troels W. Kjær ${ }^{\S}$, Ernesta M. Meintjes ${ }^{\dagger}$, \\ Christopher D. Molteno**, Joseph L. Jacobson", Sandra W. Jacobson", and Helge B.D. Sorensen*
}

\begin{abstract}
In this study event-related potentials (ERPs) were used to investigate the effects of prenatal alcohol exposure on response inhibition identified during task performance. ERPs were recorded during a auditory Go/No Go task in two groups of children with mean age of 12.8 years (11 years to 14.7 years): one diagnosed with fetal alcohol syndrome (FAS) or partial FAS (FAS/PFAS; $n=12$ ) and a control group of children of same age whose mothers abstained from alcohol or drank minimally during pregnancy $(n=11)$. The children were instructed to push a button in response to the Go stimulus and not to press the button when the No Go stimulus were heard.

Task performance accuracy did not differ between the two groups, however differences were observed in the ERP components: P2, N2, and P3. The P2 amplitude were larger for Go trials in both groups. The FAS/PFAS group showed slower $\mathrm{N} 2$ response to Go trials, suggesting a less efficient early classification of the stimulus. P3 showed larger amplitudes to No-Go vs. Go in both groups.
\end{abstract}

The study has provided new evidence for inhibition deficits in FAS/PFAS subjects identified by ERPs.

\section{INTRODUCTION}

Mothers failing to abstaining from alcohol during pregnancy will expose their child to alcohol, in which case the child is said to have had prenatal exposure to alcohol (PEA). Fetal Alcohol Syndrome (FAS), one of several disorders related to PEA, was first described in 1973 as a set of facial anomalies (short palpebral fissures, flat midface, thin upper lip, and a flat or smooth philtrum), accompanied by prenatal and/or postnatal growth retardation and cognitive/behavioral issues [1]. Children showing typical facial dysmorphic and abnormalities in one of the other domains (growth or central nervous system structure or function) is diagnosed with partial FAS (PFAS) [2]. The term Fetal Alcohol Spectrum Disorder (FASD) describes the continuum of adverse effects of alcohol on the developing human, with FAS being in the severe end. Despite the absence of some of the characteristic dysmorphic features, the severity of the cognitive deficits associated with PFAS can be comparable to those seen in FAS, therefore these two groups are often combined for analyses related to cognitive and executive function deficits [3].

It is has been suggested that deficits in response inhibition, a key aspect of executive function that refers to the ability to

* Department of Electrical Engineering, Technical University of Denmark, Kgs. Lyngby, Denmark ${ }^{\dagger}$ Department of Human Biology, Faculty of Health Sciences, University of Cape Town, Cape Town, South Africa ${ }^{*}$ Department of Oral Medicine, University of Copenhagen, Copenhagen, Denmark ${ }^{5}$ Department of Clinical Neurophysiology NF-3063, Rigshospitalet, Copenhagen, Denmark Department of Psychiatry and Behavioral Neurosciences, Wayne State University School of Medicine, Detroit, Michigan ${ }^{* *}$ Department of Psychiatry, Faculty of Health Sciences, University of Cape Town, Cape Town, South Africa selectively respond and attend to non-targets while inhibiting responses to targets, may be related to PEA [3]. Event-related Potentials (ERP), which is derived from Electroencephalography (EEG) recordings, is a class of evoked potentials that reflect task-specific changes in the electric activity in the brain. ERPs can be particular useful for characterizing brain activity in different experimental situations, since the ERP component amplitude $(\mu \mathrm{V})$ and latency (ms) vary with task demands and stimuli.

The ERP components that are most likely to reflect sensory and cognitive processes associated with task demands are the P2-N2-P3 complex. In the auditory domain the function of the ERP component P2, a positive wave typically occurring in the interval $150 \mathrm{~ms}$ to $250 \mathrm{~ms}$ after stimulus onset, is quite unknown, however it is though to play a role in stimulus classification and in inhibition processes [4]. Typically evoked at latency $200 \mathrm{~ms}$ to $350 \mathrm{~ms}$ is the second negative wave, N2, also thought to be related to the cognitive process of stimulus identification and distinction. Finally P3 (also known as P300), the most investigated ERP component, is a positive voltage deflection in the interval $250 \mathrm{~ms}$ to $600 \mathrm{~ms}$ generally thought to reflect the level of attention and task demand [5].

Recently Burden et al. 2009 [3] have investigated effects of FAS/PFAS on response inhibition in children by studying eventrelated potentials (ERP) using a visual Go/No-Go paradigm. It was found that there was a significant difference between the FAS/PFAS group and the normal controls in: P2 latency, N2 amplitude, P3 amplitude, and late slow wave (LSW). Kaneko et al. 1996 [6] found significant longer P300 latencies for patients with FASD.

This study sets out to investigate the above mentioned ERP components in patients with FASD in response to auditory stimuli and compare the results with those found in the excellent study by Burden et al. [3], in which a visual stimuli Go/No-Go paradigm was used on a subset of the subjects available for the present study. For the purpose of comparability the methods used in this paper has been chosen to resemble the methods used by Burden et al. as close as possible.

\section{Methods}

\section{A. Participants}

The participants used in this study were recruited from the Cape Coloured (mixed ancestry) community in Cape Town, South Africa. A part of the South African population known to have a very high prevalence of FAS/PFAS [7]. The subjects were recruited from two groups: The older siblings of children in the Cape Town Longitudinal Cohort Study [8]. Children 
selected based on a screening undertaken at an elementary school in a nearby rural section of Cape Town, where there is a very high incidence of alcohol abuse among local farm workers [9]. The diagnosis of FAS/PFAS in the subjects were made by one of three expert dysmorphologists (HEH, LKR, NK; see [8]) based on the revised Institute of Medicine criteria [2]. Only children who met the diagnostic criteria for FAS or PFAS or were exposed to alcohol no more than minimal during pregnancy (controls) were included in the study.

A total of 24 FAS/PFAS children and 20 control nonexposed right-handed children participated including nine of children with FAS/PFAS and seven of the control children that also participated in the visual study [3]. However, ERP from 12 of the children with FAS/PFAS and 9 of the normal control children were not available for analysis due to technical difficulties, poorly formed ERP, inability to perform the task, or uncooperative behavior of the child. The remaining 12 children with FAS/PFAS and 11 normal controls were included in the analyses. To asses the participants perceptual hearing threshold of the participants an audiology screening was conducted prior to the auditory Go/No-Go task.

\section{B. Go/No-Go Task}

In the auditory Go/No-Go task the child was instructed to press a button with the right index finger as soon as possible in response to every $1000 \mathrm{~Hz}$ tone (Go trials) and not to press the button when the $2000 \mathrm{~Hz}$ tone was heard (NoGo trial). The auditory stimuli had a duration of $500 \mathrm{~ms}$ and were presented with an inter-stimulus-interval of $4500 \mathrm{~ms}$ with silence in between. Go and No-Go stimuli were presented randomly within 7 consecutive blocks of 50 trials each (35 Go and 15 No-Go) resulting in a total of 350 trials. Each block was preceded by a 1 minute rest period.

\section{Electroencephalography Recording}

Continuous eletroencephalography (EEG) data were recorded using a 128-channel Electro Geodesics (EGI) Net Amps 200 system with a HydroCel Geodesic Sensor Net (HCGSN). The EEG signals were digitized at a rate of $250 \mathrm{~Hz}$. In this study only electrodes matching those of the international 10-20 was considered, that is anterior-frontal (Fp1, Fp2), frontal (F3, F4, F7, F8, Fz), central (C3, C4), parietal (P3, P4, Pz), and occipital (O1, O2, Oz). In addition left and right mastoids (LM, $\mathrm{RM}$ ), bipolar electrodes placed at the canthi of the right eye, and bipolar electrodes placed above and below the eye were recorded for re-referencing and artifact detection and correction. All electrodes were recorded with $\mathrm{Cz}$ as reference.

\section{ERP Data Analysis}

ERPs were derived offline using routines developed in MATLAB 2010A (MathWorks, USA). All trials were rereferenced to average mastoids and electrooculogram (EOG) corrected using the eye movement correction procedure (EMCP) described in [10] prior to averaging. In the EMCP the event related eye movement is estimated and subtracted from the EOG channel before using it for correcting the EEG data. For correcting the EEG data a correction factor $K$ is calculated by solving $K$, using linear regression model with least squares estimator, in the equation,

$$
V_{t}-\bar{V}_{t}=K\left(E O G_{t}-\overline{E O G}_{t}\right)
$$

Here $V_{t}$ is the EEG potential at time point $t, \bar{V}_{t}$ is the ERP potential (average of stimuli time locked EEG trials), $E O G_{t}$ is the raw EOG channel data at time point t, and $\overline{E O G}_{t}$ is the averaged trial EOG derived by time locking the EOG for each trial to the eliciting event (Go/No-Go stimuli). All validated (cf. next paragraph) trial data is used in the regression. The corrected EEG is calculated using,

$$
\text { Corrected } E E G=\operatorname{RawEEG}-K(\operatorname{Raw} E O G)
$$

The EOG channel data was digitally bandpass filtered with lower and higher cutoff frequencies of $0.53 \mathrm{~Hz}$ and $13 \mathrm{~Hz}$ before being used in the EMCP.

Prior to EOG correction the EEG data were digitally bandpass filtered with lower and higher cutoff frequencies of $0.796 \mathrm{~Hz}$ and $30 \mathrm{~Hz}$. Trials with channels exceeding $\pm 150 \mu \mathrm{V}$ or with extreme wave morphology were rejected as well as trials with incorrect responses (button press after No-Go stimulus or lacking button press after Go stimulus). For the EOG channel trials exceeding $\pm 1000 \mu \mathrm{V}$ were rejected. Individual ERP averages of the remaining EOG-corrected trials were computed with segments consisting of a baseline period of $100 \mathrm{~ms}$ prior to stimulus onset followed by a $1500 \mathrm{~ms}$ post stimulus recording. Peak detection was used to identify the amplitude and latency of the following three ERP components: $\mathrm{P} 2$, a positive deflection in the interval $100 \mathrm{~ms}$ to $280 \mathrm{~ms}$ from stimulus onset; N2, a negative deflection in the interval $200 \mathrm{~ms}$ to $360 \mathrm{~ms}$; and P3, a positive deflection in the interval $280 \mathrm{~ms}$ to $600 \mathrm{~ms}$. The intervals were chosen in collaboration with a board certified clinical neurophysiologist Troels W. Kjær (TWK) such that the components in the individual subject ERPs would all be detected. The amplitude measures reported for these components represent mean activity surrounding the peak ( $\pm 20 \mathrm{~ms})$.

\section{E. Statistical Analysis}

Mixed design repeated measures analyses of variance (ANOVA) was used to assess behavioral task performance for correct responses and mean reaction time (RT) in separate 2 (group: FAS/PFAS, controls) $\times 2$ (condition: Go, No-Go) analyses, with group as between-subject factor and condition as the within-subjects factor. For ERPs, repeated measures ANOVAs were used to measure peak amplitude $(\mu \mathrm{V})$ and latency (ms) effects for P2, N2, and P3, focusing on the 6 electrodes where effects were maximal (F3, Fz, F4, C3, Cz, C4) in a $2 \times 2 \times 3 \times 2$ (group; condition; hemisphere: left, midline, right; region: frontal, central) design, with group as the betweensubjects factor. All statistical analyses were conducted using MATLAB 2010A Statistics Toolbox. 
Table I

DEMOGRAPHIC CHARACTERISTICS BY EXPOSURE GROUPS

\begin{tabular}{|c|c|c|c|c|c|}
\hline & \multicolumn{2}{|c|}{ FAS/PFAS $(n=12)$} & \multicolumn{2}{|c|}{ Controls $(n=11)$} & \multirow[b]{2}{*}{$F$} \\
\hline & $\mathrm{M}(\mathrm{SD})$ or $\mathrm{n}(\%)$ & Range & $\mathrm{M}(\mathrm{SD})$ or $\mathrm{n}(\%)$ & Range & \\
\hline \multicolumn{6}{|l|}{ Child characteristics } \\
\hline Gender (number of females) & $8(66.7)$ & - & $4(36.4)$ & - & \\
\hline Age at test (years) & $13.0(1.13)$ & $11.3-14.6$ & $12.8(1.3)$ & $11.0-14.7$ & $<1$ \\
\hline Lead $(\mathrm{Pb} ; \mu \mathrm{g} / \mathrm{dl})$ & $5.0(2.4)$ & $2-12$ & $7.1(3.3)$ & $3-12$ & 1.80 \\
\hline \multicolumn{6}{|l|}{ Maternal characteristics } \\
\hline Age at delivery (years) & $25.2(5.5)$ & $17.2-33.1$ & $26.7(6.2)$ & $14.9-40.1$ & $<1$ \\
\hline Socioeconomic status & $17.4(6.6)$ & $9.5-29$ & $19.3(10.0)$ & $6-37.5$ & $<1$ \\
\hline Education (years) & $7.45(3.0)$ & $0-10$ & $8.2(1.8)$ & $6-12$ & $<1$ \\
\hline Marital status (number married) & $5(42.0)$ & - & $6(54.5)$ & - & \\
\hline Cigarettes during pregnancy (per day) & $11.0(8.8)$ & $0-30$ & $9.8(7.0)$ & $0-20$ & $<1$ \\
\hline
\end{tabular}

Table II

Means (Standard Deviations) For Behavioral Performance

\begin{tabular}{lcc}
\hline & FAS/PFAS $(n=12)$ & Controls $(n=11)$ \\
\hline Accuracy & $95.9(5.5)$ & $96.4(6.7)$ \\
Correct Go $(\%)$ & $85.1(11.5)$ & $85.8(9.1)$ \\
$\quad$ Correct No-Go $(\%)$ & $634(624 ; 643)$ & $613(602 ; 625)$ \\
Reaction time & & \\
$\quad$ Correct Go $(\mathrm{ms})$ &
\end{tabular}

\section{RESULTS}

\section{A. Background Characteristics}

In Table I the demographic characteristics of child and the primary caregiver. The FAS/PFAS group and the controls group did differ in case of the gender and the lead levels. None of the mothers reported use of cocaine, marijuana, hashish, methaqualone ("mandrax"), or other drugs during pregnancy, and no significant $(\alpha=0.01)$ group differences were found in number of cigarettes smoked per day.

\section{B. Behavioral Performance}

For the sample as a whole, there was a greater accuracy (\% correct) for Go versus No-Go trials, $M=96.1 \%$ (Go) vs. $M=85.4 \%$ (No Go), $t=38.5, p<0.05$. This indicates that it is more difficult to inhibit a response than to execute the button press, as expected [3]. There was no significant difference in Go reaction time between the groups $(t=-2.65, p<0.05)$. The overall performance was high as seen in Table II.

\section{ERP Measures}

In Figure 1 the grand ensemble ERPs for the two groups and the two conditions are shown. In Table III the latencies and amplitudes calculated using a full automatic system for the regions where the effects were identified as maximal.

The P2 amplitude showed a condition effect, $F_{1,21}=5.88$, $p=0.02$, indicating an overall larger $\mathrm{P} 2$ to Go vs. No-Go. A hemisphere $\times$ region interaction was also found, $F_{2,42}=16.94$, $p<0.05$, and follow-up test indicated that there was an overall difference between midline-frontal and midline central, $M=$ $4.36 \mu \mathrm{V}$ vs. $M=8.67 \mu \mathrm{V}, F_{1,90}=27.75, p<0.05$. An central hemisphere effect was also evident with highest amplitudes at the midline $(M=8.6 \mu \mathrm{V})$ compared to left $(M=5.0 \mu \mathrm{V})$ and right $(M=5.3 \mu \mathrm{V})$.

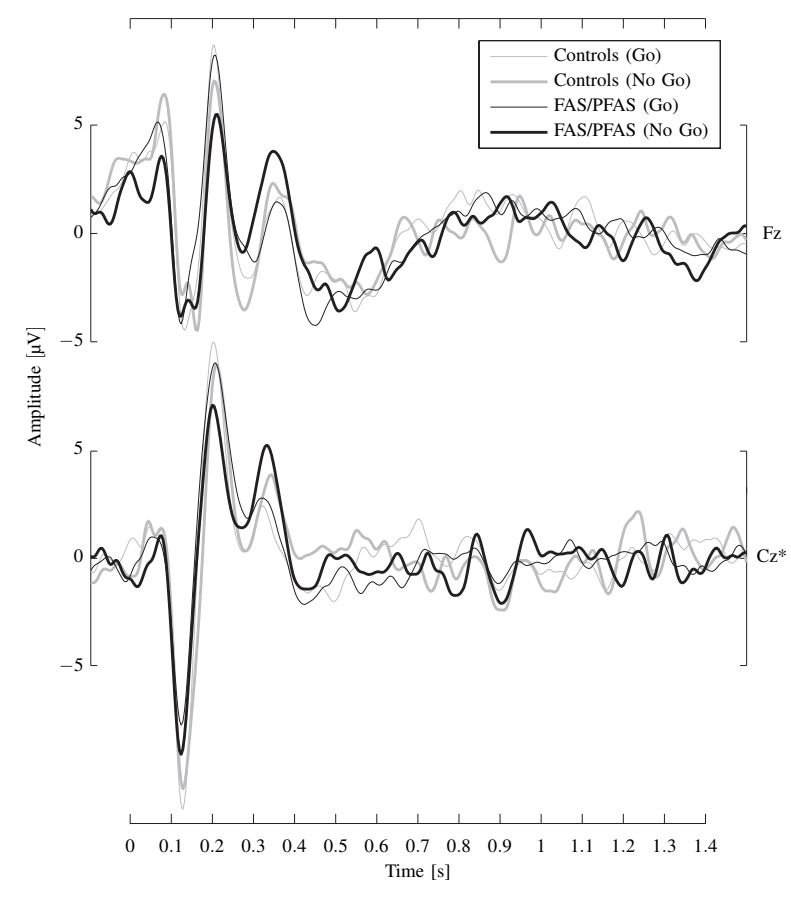

Figure 1. Stimulus-locked grand means at $\mathrm{Fz}, \mathrm{Cz}^{*}(\mathrm{Cz}$ was used as reference, E55 shown here) for controls Go (thin gray), controls No-Go (bold gray), FAS/PFAS go (thin black), and FAS/PFAS No-Go (bold black). Baseline period $(100 \mathrm{~ms})$ represents $100 \mathrm{~ms}$ prior to stimulus onset.

Table III

ERP COMPONENT MEANS AND STANDARD ERRORS. AMPLITUdES FROM FZ AND LATENCIES FROM C3.

\begin{tabular}{cccccc}
\hline & \multicolumn{2}{c}{ FAS $(n=12)$} & & \multicolumn{2}{c}{ Controls $(n=11)$} \\
\cline { 2 - 3 } \cline { 5 - 6 } & Go & No Go & & Go & No Go \\
\hline P2 & & & & \\
Latency $(\mathrm{ms})$ & $209(11.9)$ & $209(10.8)$ & $205(19.9)$ & $215(24.5)$ \\
Amplitude $(\mu \mathrm{V})$ & $4.9(4.3)$ & $2.8(4.4)$ & $5(3.5)$ & $4.9(3.9)$ \\
N2 & & & & \\
Latency $(\mathrm{ms})$ & $290(28.9)$ & $260(24)$ & $276(31.6)$ & $277(20.3)$ \\
Amplitude $(\mu \mathrm{V})$ & $-3.5(2.6)$ & $-4.1(2.7)$ & $-3.6(3)$ & $-4.2(3.2)$ \\
P3 & & & & \\
Latency $(\mathrm{ms})$ & $338(27.2)$ & $318(16.4)$ & $333(33.6)$ & $330(25.9)$ \\
Amplitude $(\mu \mathrm{V})$ & $2(2.1)$ & $4.2(3.6)$ & $1.8(2.9)$ & $2.6(3.3)$ \\
\hline
\end{tabular}


For P2 latencies there was a main effect for for hemisphere, $F_{2,21}=4.10, p=0.027$, indicating that the latencies were slower for the left hemisphere $(M=206.7 \mathrm{~ms})$ compared to midline $(M=200.9 \mathrm{~ms})$.

The N2 amplitudes had a significant hemisphere $\times$ region interaction, $F_{2,42}=7.93, p=0.017$. Post hoc tests revealed that there were greater $\mathrm{N} 2$ (more negative) in the frontal sites versus the central sites for left and right hemispheres. In the central region there was a more negative $\mathrm{N} 2$ at the right hemisphere versus the midline.

For N2 latency there was a group $\times$ condition interaction effect. A significant difference between the two groups for the Go trials was found, $F_{1,136}=6.25, p=0.0136, M_{\text {control }}=$ $270 \mathrm{~ms}$ vs $M_{F A S D}=281 \mathrm{~ms}$. Furthermore a difference between Go and No-Go trials in the FAS/PFAS group was evident, $F_{1,142}=19.41, p<0.005, M_{F A S D, g o}=281 \mathrm{~ms}$ vs $M_{F A S D, n g}=$ $263 \mathrm{~ms}$, an effect completely absent in the normal control group.

For the P3 amplitude there was a main condition effect, $F_{1,21}=9.79, p=0.005$, indicating higher P3 amplitude to NoGo vs Go. A main region effect, $F_{1,21}=7.03, p<0.05$, was also found, which indicated a higher overall P3 amplitude for the frontal vs central sites, $M=2.78 \mu \mathrm{V}$ vs $M=1.87 \mu \mathrm{V}$. For the P3 latency there was an overall region effect, $F_{1,21}=7.18$, $p=0.014$, that indicated slower latencies at the frontal sites.

\section{DISCUSSION}

This study is one of few to identify deficits in response inhibition in children with FAS/PFAS using ERPs. Both the normal controls and the FAS/PFAS groups performed well in the Go/No Go task which was expected as the task was designed to be relatively easy to minimize group differences. As expected the subjects made more errors in the No Go trials than in the Go trials [3], [11].

However several effects became clear in the ERP analyses. $\mathrm{P} 2$, which is thought to play a role in early stimulus classification and possible response inhibition, showed a larger amplitude in response to Go vs. No-Go in both groups, which could signify an early discrimination of the stimulus. In [3] this effect was only seen in the normal controls, however this difference could originate from the different stimulus paradigm (auditory vs. visual).

On N2 the longer latencies to Go trials in the FAS/PFAS was unexpected. Davis et al. [11] contrarily found a shorter N2 latency for children (6years to 7 years) in response to Go compared to No Go. However, for adults Davis et al. found the opposite which could indicate that different processes is being used at different ages. The children in the control group did not show any differences in Go vs No-Go. These results may indicate that at this age (13years) the latencies start to shift. The lack of amplitude differences in relation to condition was unexpected as this is normally a clear marker for response inhibition.

Both groups showed as expected a larger P3 to No-Go, which likely indicates an increased allocation of attention when actively inhibiting a prepotent tendency to respond. This link between the P3 amplitude and response inhibition has been documented repeatedly in the literature [12], [5], [3].

In summary this study has indicated several ERPs results in response to auditory stimuli similarly to those found by Burden et al. 2009 [3] and added to the knowledge about the specific changes in ERP caused by FASD.

\section{Future Work}

Inclusion of gender in the analysis. Investigation of more advanced peak detection methods and use of advanced tracking methods such as the Kalman filtering used in [13] could improve time series analyses like those done in this study. Furthermore it could be interesting to investigate the time-frequency structure of ERPs of patients with FASD.

\section{ACKNOWLEDGEMENTS}

Supported by a Fogarty International Research Collaboration Award from the National Institutes of Health [R03 TW007030 to SWJ]; a Focus Area grant [FA2005040800024 to EMM] from the National Research Foundation of South Africa; the South African Research Chairs Initiative of the Department of Science and Technology and National Research Foundation of South Africa; Medical Research Council of South Africa; a Children's Bridge grant from the Office of the President of Wayne State University to JLJ; and seed money grants from the University of Cape Town and the Joseph Young, Sr., Fund from the State of Michigan. The dysmorphology assessments were conducted in conjunction with the NIH/National Institute on Alcohol Abuse and Alcoholism Collaborative Initiative on Fetal Alcohol Spectrum Disorder [U01-AA014790, U24AA014815]

\section{REFERENCES}

[1] K. L. Jones and D. W. Smith, "Recognition of the fetal alcohol syndrome in early infancy," The Lancet, vol. 302, no. 7836, pp. 999 - 1001, 1973

[2] H. Hoyme et al., "A practical clinical approach to diagnosis of fetal alcohol spectrum disorders: clarification of the 1996 institute of medicine criteria," Pediatrics, vol. 115, no. 1, p. 39, 2005.

[3] M. J. Burden et al., "The effects of fetal alcohol syndrome on response execution and inhibition: An event-related potential study," Alcoholism: Clinical and Experimental Research, vol. 33, no. 11, pp. 1994-2004, 2009.

[4] K. E. Crowley and I. M. Colrain, "A review of the evidence for p2 being an independent component process: age, sleep and modality," Clinical Neurophysiology, vol. 115, no. 4, pp. 732 - 744, 2004.

[5] K. H. Chiappa, Evoked Potentials In Clinical Medicine, 3rd ed. Lippincott Raven, 1997.

[6] W. M. Kaneko et al., "Auditory event-related potentials in fetal alcohol syndrome and down's syndrome children," Alcoholism: Clinical and Experimental Research, vol. 20, no. 1, pp. 35-42, 1996.

[7] P. May et al., "Epidemiology of fetal alcohol syndrome in a south african community in the western cape province," Am J Public Health, vol. 90, no. 12 , pp. 1905-1912, 2000.

[8] S. W. Jacobson et al., "Impaired eyeblink conditioning in children with fetal alcohol syndrome," Alcoholism: Clinical and Experimental Research, vol. 32, no. 2, pp. 365-372, 2008.

[9] N. C. Dodge et al., "Prenatal alcohol exposure and interhemispheric transfer of tactile information: Detroit and cape town findings," Alcoholism: Clinical and Experimental Research, vol. 33, no. 9, pp. 1628-1637, 2009.

[10] G. Gratton, M. G. H. Coles, and E. Donchin, "A new method for offline removal of ocular artifact," Electroencephalography and Clinical Neurophysiology, vol. 55, no. 4, pp. 468 - 484, 1983.

[11] E. P. Davis et al., "The x-trials: Neural correlates of an inhibitory control task in children and adults." Journal of Cognitive Neuroscience, vol. 15, no. 3, pp. $432-443,2003$.

[12] A. Tekok-Kilic, J. L. Shucard, and D. W. Shucard, "Stimulus modality and go/nogo effects on $\mathrm{p} 3$ during parallel visual and auditory continuous performance tasks," Psychophysiology, vol. 38, no. 3, pp. 578-589, 2001.

[13] E. Vedel-Larsen et al., "A comparative study between a simplified kalman filter and sliding window averaging for single trial dynamical estimation of event-related potentials," Computer Methods and Programs in Biomedicine, vol. 99, no. 3, pp. 252 - 260, 2010. 